\title{
Pour une histoire intégrante. La construction de la mémoire dans une société diversifiée
}

\section{Gérard Bouchard}

Volume 66, numéro 3-4, hiver-printemps 2013

URI : https://id.erudit.org/iderudit/1025531ar

DOI : https://doi.org/10.7202/1025531ar

Aller au sommaire du numéro

Éditeur(s)

Institut d'histoire de l’Amérique française

ISSN

0035-2357 (imprimé)

1492-1383 (numérique)

Découvrir la revue

Citer cet article

Bouchard, G. (2013). Pour une histoire intégrante. La construction de la mémoire dans une société diversifiée. Revue d'histoire de l'Amérique française, 66(3-4), 291-305. https://doi.org/10.7202/1025531ar
Résumé de l'article

La science historique, on le sait, a toujours eu partie liée avec l'actualité ; elle ne se construit jamais à distance de ses soubresauts. La société québécoise en offre présentement quelques exemples éloquents. Ainsi, depuis quelques années, une controverse s'est élevée au sujet de l'enseignement de l'histoire nationale ; certains voudraient lui assigner une fonction à dominante civique, alors que d'autres insistent sur sa vocation d'éveilleuse de la conscience nationale. En parallèle, une autre controverse a mis en cause la survie de la culture québécoise dans un contexte de diversité ethnoculturelle. L'orientation pluraliste préconisée par un grand nombre de citoyens (et mise en forme dans l'interculturalisme québécois) est accusée de compromettre la continuité culturelle du Québec francophone, contraignant en quelque sorte ses membres à renoncer à leur mémoire, d'oublier ce qu'ils sont. De la même façon, le débat sur les valeurs dites nationales présente couramment les valeurs universelles comme impropres à nourrir une véritable identité ; on serait ici irrémédiablement confronté à la dichotomie particularisme-universalisme. Dans chacun de ces cas (et d'autres qui sont brièvement abordés dans le texte), on observe que deux sphères ou deux entités sont posées comme antinomiques. Cet article voudrait montrer que c'est une erreur, qu'en réalité on est en présence non pas d'une contradiction mais d'une complémentarité. 
La rédaction de la Revue d'histoire de l'Amérique française est fière de publier le texte de la conférence d'ouverture du $66^{e}$ Congrès de l'Institut d'histoire de l'Amérique française, tenu à Rimouski en octobre 2013.

|

\section{Pour une histoire intégrante. La construction de la mémoire dans une société diversifiée}

GÉRARD BOUCHARD

Université du Québec à Chicoutimi

RÉsumé - La science historique, on le sait, a toujours eu partie liée avec l'actualité; elle ne se construit jamais à distance de ses soubresauts. La société québécoise en offre présentement quelques exemples éloquents. Ainsi, depuis quelques années, une controverse s'est élevée au sujet de l'enseignement de l'histoire nationale; certains voudraient lui assigner une fonction à dominante civique, alors que d'autres insistent sur sa vocation d'éveilleuse de la conscience nationale. En parallèle, une autre controverse a mis en cause la survie de la culture québécoise dans un contexte de diversité ethnoculturelle. L'orientation pluraliste préconisée par un grand nombre de citoyens (et mise en forme dans l'interculturalisme québécois) est accusée de compromettre la continuité culturelle du Québec francophone, contraignant en quelque sorte ses membres à renoncer à leur mémoire, d'oublier ce qu'ils sont. De la même façon, le débat sur les valeurs dites nationales présente couramment les valeurs universelles comme impropres à nourrir une véritable identité; on serait ici irrémédiablement confronté à la dichotomie particularisme-universalisme. Dans chacun de ces cas (et d'autres qui sont brièvement abordés dans le texte), on observe que deux sphères ou deux entités sont posées comme antinomiques. Cet article voudrait montrer que c'est une erreur, qu'en réalité on est en présence non pas d'une contradiction mais d'une complémentarité.

ABstract - As we know, historical science has always resonated with the present; it never shields itself from its shifts and turns. In this regard, the Québec society offers striking examples. For instance, over the last decade, a controversy broke out over the teaching of national history. Some would like it to serve a civic purpose while others emphasize its function as a source of national consciousness. In a different but closely related vein, another controversy involves the future of the Québec culture in a context of ethno-cultural diversification. The pluralist orientation advocated by a number of citizens (and implemented in Québec inter- 
culturalism) is said to threaten -if not to jeopardize-- the cultural continuity of Francophone Québec, thus compelling its members to renounce their identity, to forget who they are. Likewise, the debate over national values depicts the universal values as unsuited to feed a true identity. Here, one is faced with the particularism-universalism dichotomy. In each of these cases (and a few others that are briefly touched on in the text), two spheres are cast as antinomic. Yet, this article aims to show that this is a mistake, that deep down lies a complementarity rather than a contradiction.

\section{DIVERSITÉ ET PLURALISME}

En Occident comme ailleurs dans le monde, un très grand nombre de nations ont tiré une leçon des horreurs de la première moitié du $\mathrm{XX}^{\mathrm{e}}$ siècle, à savoir les deux guerres mondiales, les despotismes, les fascismes, les génocides, la fin des colonialismes. Cette leçon, c'est le pluralisme, c'est-à-dire le respect de la diversité et des droits de la personne: au lieu de briser ou d'opprimer la différence, on s'emploierait désormais à l'accepter, à l'intégrer. À cela s'est ajoutée une nouvelle prise de conscience des impératifs de la démocratie.

Ce nouvel esprit, qui a connu un essor spectaculaire au lendemain de la Deuxième Guerre mondiale, a rendu possible l'émancipation des minorités de toutes sortes: raciales, linguistiques, religieuses, sexuelles, et le reste. Il a puissamment aidé, notamment, à l’amélioration de la condition féminine.

C'est sur cet arrière-plan qu'il faut analyser la hausse de l'immigration consécutive à la mondialisation et les effets qu'elle a exercés sur les sociétés d'accueil. À cause du pluralisme, il n'était plus possible de pratiquer l'exclusion ou l'assimilation à l'endroit de l'étranger comme il était d'usage jusqu'alors. Parallèlement, l'homogénéité cessait d'être le prisme devant servir de définition et même d'idéal à la nation. En conséquence, de vieux codes culturels, de robustes configurations mythiques et de profonds enracinements identitaires et mémoriels se trouvaient remis en question.

Sous le choc, certaines sociétés ont choisi de se durcir, refoulant les nouveaux apports symboliques dans des marges, alors que d'autres sociétés, au contraire, ont cherché à mettre en œuvre des réaménagements afin d'intégrer ces nouveaux apports. Le Québec fait partie de ce deuxième groupe de sociétés et je vais lui consacrer la suite de cette réflexion. 


\section{L'AMÉNAGEMENT DE LA DIVERSITÉ: LA MANIÈRE QUÉBÉCOISE}

C'est à partir des années 1950 et surtout avec la Révolution tranquille que les Francophones québécois ont commencé à se représenter vivement la diversité de leur société et à en tirer les conclusions. Trois facteurs, principalement, y ont contribué. Premièrement, la chute brutale de la fécondité réactivait l'inquiétude pour la survivance culturelle et favorisait une nouvelle prise de conscience de l'Autre, en l'occurrence les minorités déjà en place au Québec. Deuxièmement, cette perception était accentuée par la croissance de l'immigration et la diversification des pays de provenance des nouveaux arrivants. Troisièmement, l'émergence d'une identité québécoise, largement délestée de la vieille appartenance pancanadienne, installait les anciens Canadiens français du Québec dans une position de majorité, ce qui, inévitablement, mettait davantage en relief la présence des minorités.

C'est dans ces conditions que le Québec s'est mis à la recherche d'une nouvelle définition de lui-même et, plus précisément, d'un modèle de gestion de sa diversité. Dans un premier temps, le multiculturalisme canadien a été officiellement rejeté en 1971 par le premier ministre Robert Bourassa dans une lettre adressée au premier ministre canadien, Pierre E. Trudeau:

Le Québec n’adopte pas, au niveau du principe du multiculturalisme, l'approche de votre gouvernement... Le Québec doit assumer le rôle de premier responsable sur son territoire de la permanence de la langue et de la culture française ${ }^{1}$.

Par la suite, le gouvernement québécois, à l'aide de chercheurs universitaires et autres, s'est appliqué à développer une formule originale qui allait devenir l'interculturalisme québécois. Il est important de rappeler les idées maîtresses qui, dès le départ, ont servi de fondement à ce modèle.

- C'est d'abord le rejet à la fois des formules d'assimilation et des formules de fragmentation.

- C'est ensuite la perception d'un rapport fondamental entre une majorité francophone et des minorités ethnoculturelles. Ce rapport majorité-minorités était dès lors posé comme un paramètre structurel de la réalité ethnoculturelle québécoise.

1. Lettre du 11 novembre 1971, citée dans Micheline Labelle, «Les intellectuels québécois face au multiculturalisme: hétérogénéité des approches et des projets politiques », Canadian Ethnic Studies, 40, 1-2 (2008): 39 . 
- C’est, en troisième lieu, la recherche d'un équilibre entre les nécessités du droit et les impératifs de l'identitaire.

- C'est, enfin, l'obligation de gérer décemment, en accord avec l'orientation pluraliste, le rapport majorité-minorités. On en faisait la finalité globale de l'interculturalisme².

Suivant ces directions, le Québec a pu élaborer un modèle original dont s'est inspiré récemment le Conseil de l'Europe. Dans le cours d'une large consultation effectuée en 2006-2007 auprès de ses 47 États membres (donnant suite au Sommet de Varsovie en 2005), ces derniers étaient interrogés sur le meilleur modèle à promouvoir en matière de relations interethniques. Or, tous ces pays en sont arrivés à un consensus en trois points : a) le rejet du multiculturalisme, associé au risque de fragmentation et perçu comme nuisible à la cohésion sociale, b) le rejet de l'assimilation, à cause de la violation des droits individuels à laquelle il conduit, c) le choix de l'interculturalisme comme voie médiane, comme modèle d'équilibre et d'équité. La consultation faisait également ressortir que ce modèle retenait ce qu'il y a de meilleur dans le multiculturalisme (la sensibilité à la diversité) et dans le républicanisme (la sensibilité à l'universalité des droits) $)^{3}$.

Dans divers pays européens, on tient de plus en plus l'interculturalisme comme la formule de rechange du multiculturalisme, maintenant tombé en défaveur là-bas. Pourquoi cet intérêt qui déborde le cadre européen et s'étend en Amérique latine et en Asie, tout particulièrement au Japon? La réponse tient dans les équilibres que l'interculturalisme invite à négocier entre des impératifs qui se présentent souvent sous la forme d'antinomies. La pensée québécoise, jusqu'à récemment, s'est montrée exemplaire à cet égard. Elle s'est caractérisée, en particulier, par un rejet des fausses antinomies ou dichotomies. J'en donne quatre exemples.

\section{DE FAUSSES ANTINOMIES}

\section{L'ethnique et le civique}

Selon une perception courante que l'on observe en particulier chez les opposants au pluralisme culturel ${ }^{4}$, le juridique et le culturel ne feraient pas bon ménage. Les règles froides, aveugles, du droit exerceraient un

2. Sur ce sujet, voir Gérard Bouchard, L'interculturalisme. Un point de vue québécois (Montréal, Boréal, 2012), chap. 2.

3. Conseil de l'Europe, Comité des Ministres, $118^{\mathrm{e}}$ session, Livre blanc sur le dialogue interculturel: "Vivre ensemble dans l'égale dignité» (2008).

4. Voir à ce sujet un survol dans G. Bouchard, op. cit., 109-154. 
effet corrosif sur les diverses dimensions de la culture, à commencer par l'identité nationale. Au cours des dernières décennies, cette antinomie s'est exprimée principalement sous la forme de la polarité civique-ethnique, selon laquelle il y aurait des nations entièrement vouées au respect $\mathrm{du}$ droit individuel alors que d'autres instaureraient la prédominance d'une majorité ethnique, homogène, qui gouvernerait en fonction de ses intérêts, soucieuse en priorité de préserver ses traditions et son identité, ce qui placerait les minorités devant deux options: s'assimiler ou vivre dans l'exclusion.

En fait, il s'agit là au mieux d'un type idéal, au pire d'une caricature. Telle que décrite la plupart du temps, la nation civique est une utopie. Aucune société ne peut faire l'économie d'un fondement symbolique composé de valeurs, d'idéaux, de références mémorielles et d’appartenance partagés. C'est ce fondement symbolique qui soutient tout lien social et permet à une société de faire des choix, de construire des consensus, de se donner des orientations, de se mobiliser autour de projets de changement, de se redresser après une crise, etc. Ce serait donc instituer un faux procès de xénophobie que de condamner les attaches ou les aspirations identitaires au nom du pluralisme.

Quant à la nation dite ethnique, on peut la rencontrer sous des formes tribales ou sous la forme de minorités nationales, mais en Occident tout au moins, on connaît très peu d'États-nations démocratiques qui souscrivent à sa définition. Le modèle courant de l'État-nation, c'est toujours un alliage de culture et de droit calibré et agencé de différentes manières ${ }^{5}$. De ce point de vue, le Québec se situe pleinement dans une position d'équilibre. On le voit à la façon dont, entre les années 1960 et le début $\mathrm{du} \mathrm{XXI}{ }^{\mathrm{e}}$ siècle, il a su marier une forme vigoureuse de nationalisme avec un authentique libéralisme, une performance collective qui a peu de précédents en Occident. Le Québec est ainsi parvenu à cultiver une forte identité tout en s'immergeant dans les formes les plus avancées de la modernité.

Enfin, pour qui douterait de la capacité du droit d'accueillir les aspirations collectives d'ordre culturel, je rappellerai l'exemple de la loi 101, une initiative destinée à assurer la survie de la francophonie québécoise. Même la Cour suprême du Canada - qui n'est pas précisément suspecte de com-

5. La littérature sur ce sujet est abondante. Pour une excellente démonstration prenant à témoin plusieurs pays d'Europe, voir Timothy Baycroft et Mark Hewitson, dir., What is a Nation? Europe, 17891914 (Oxford, Oxford University Press, 2006), 377 p. Dans le même ordre d'idée: Gérard Bouchard, $\mathrm{La}$ nation québécoise au futur et au passé (Montréal, VLB, 1999), 20-30. 
plaisance envers le néonationalisme québécois - a certes beaucoup amputé cette loi, mais elle n’en a pas moins reconnu la légitimité.

\section{L'universel et le singulier}

Une deuxième antinomie qui est véhiculée par la littérature et le débat public est celle qui oppose l'universel et le particulier. Dans cet esprit, chaque nation serait contrainte de se définir soit en se repliant sur ses particularismes, soit en s'en remettant à des références universelles. Encore là, il s'agit d'un faux dilemme, comme on peut le montrer à l'aide de l'exemple des valeurs. À cause de l'autorité dont jouit l'orientation pluraliste, une forte pression s'exerce désormais sur chaque nation pour qu'elle intensifie son adhésion à des valeurs universelles, par exemple: les valeurs d'égalité, de justice sociale, de liberté, de démocratie, de nonviolence. Cette pression suscite souvent un malaise au sein des nations, dans la mesure où ces valeurs dites universelles peuvent être perçues comme abstraites, froides, importées ou même imposées de l'extérieur, dans tous les cas inaptes à nourrir la singularité de l'identitaire qui se trouverait ainsi mis à l'écart.

Il faut voir les choses autrement. Prenons l'exemple de l'égalité, valeur universelle par excellence. Or, on ne remarque pas assez que cette valeur est porteuse de significations bien différentes d'une nation à l'autre. Aux États-Unis, elle réfère d'abord à l'individu et elle renvoie à l'égalité des chances en début de carrière. Pour ce qui est du point d'arrivée, de la fin de carrière, elle s'accommode de très grandes inégalités. Dans les pays scandinaves - comme au Québec - la valeur d'égalité réfère au premier chef au social et elle est beaucoup plus exigeante, s'appliquant aussi bien au point d'arrivée qu'au point de départ. En France, l'idéal d'égalité signifie d'abord l'égalité des droits civiques. On pourrait étirer ce commentaire en prenant d'autres exemples comme la liberté, la démocratie, la laïcité, et le reste.

Ce qu'il faut en retenir, c'est que les valeurs cultivées par une société ont ordinairement été forgées dans son histoire, à même des expériences vécues (positives ou négatives) qui ont produit une émotion forte. L'égalité citoyenne en France est un héritage de l'expérience d'oppression vécue sous l'Ancien Régime et des luttes révolutionnaires pour y mettre fin. Le culte de l'unité nationale aux États-Unis est en grande partie un héritage du traumatisme causé par la guerre de Sécession. L'attachement à la paix et à l'entente au sein de l'Union européenne est un héritage des atrocités de la Deuxième Guerre mondiale. Tout comme le désir d'affir- 
mation nationale au Québec est un héritage de la Conquête et de l'impérialisme britannique, etc.

Ce que je veux faire ressortir, c'est qu'au départ, les valeurs célébrées dans une société sont chargées d'un fort coefficient de particularisme. Elles entrent ensuite dans un processus de formalisation, de décantation, qui est aussi un travail de la mémoire, au terme duquel ces valeurs deviennent porteuses d'une vocation civique ou universelle. Toujours nourries de leurs racines historiques, elles parcourent un long cheminement qui en fait des valeurs qu'on peut inscrire dans des chartes et prescrire à l'ensemble des citoyens, indépendamment de leur origine ou de leur appartenance ethnoculturelle.

Pour prendre d'autres exemples tirés du contexte québécois, pensons à l'égalité homme-femme. Cet idéal est devenu très répandu à l'échelle internationale, mais il n'en est pas moins nourri ici d'un passé singulier qui le soutient et qui le ravive au besoin - même s'il s'agit d'un passé relativement récent -, l'idée ayant pris son véritable essor à partir des années 1960. Toujours dans le contexte québécois, on pourrait en dire autant de l'attachement à la laïcité, tout particulièrement la séparation de l'État et des Églises, ou de la solidarité ouvrière et des luttes syndicales.

Toute cette réflexion repose toutefois sur une exigence éthique: il est impérieux que les valeurs à promouvoir se qualifient au regard du pluralisme. Il y a donc une sélection à faire parmi les valeurs qui émergent du passé d'une société.

\section{Le pluralisme et la mémoire nationale}

Une crainte qui a été souvent formulée au cours des dernières années au Québec, et en même temps une critique qu'on a adressée au pluralisme, c'est qu'il irait à l'encontre de l'identitaire et, plus précisément, qu'il obligerait la société d'accueil à sacrifier sa mémoire (ou à "renoncer à ce qu'elle est», selon une expression courante chez ses détracteurs). Là encore, je crois qu'on est en présence d'une polarisation qui mène à de fausses perceptions de la réalité et à des débats peu fructueux. Le pluralisme impose le respect de la diversité mais non pas le relativisme. Il prescrit le respect des droits mais jamais aux dépens de ce qui constitue ce que j'ai appelé le fondement symbolique, tout particulièrement les valeurs fondamentales d'une société - je parle ici des valeurs ayant une portée civique et qui peuvent être imposées à l'ensemble des citoyens. Or, ces valeurs, comme je l'ai dit, sont, dans toute société, héritées de l'histoire dans laquelle elles continuent de baigner et de se nourrir. C’est précisé- 
ment la fonction de la mémoire que de rappeler sans cesse les racines de ces valeurs pour les maintenir vivantes. En ce sens, loin de détruire la mémoire, il arrive que les valeurs universelles se perpétuent et prospèrent grâce à elle.

C'est ce que j'appelle le processus d'historisation des valeurs, qui naissent nécessairement dans le particularisme mais qui, grâce à un travail de mémoire, accèdent ensuite à l'universalité, tout en gardant la trace de leur origine. On pourrait ainsi montrer que les valeurs universelles qui prévalent dans toutes les sociétés ont été forgées dans la singularité de leur histoire.

En d'autres mots, le progrès du pluralisme et des valeurs fondamentales se nourrit du rappel du passé - et plus précisément: des expériences les plus significatives vécues collectivement. En ce sens, il n'est pas faux de dire que le pluralisme invite non pas à renoncer à ce que l'on est mais à l'être davantage. Ce serait un non-sens que d'expurger la mémoire de ce qui, aux yeux d'une société, est le plus significatif dans son passé, de ce qui est le creuset des valeurs et des idéaux qui la définissent.

\section{L'histoire du Québec entre la nation et la citoyenneté}

Sous l'éclairage que je viens de présenter, on devrait se méfier de ce qui fonde la querelle de l'enseignement de l'histoire nationale au Québec, présentement écartelée entre le pôle de la nation et celui de la citoyenneté. Encore ici, je crois que les polarités exacerbent les orientations et créent de fausses incompatibilités qui entraînent des oppositions stériles.

La controverse qui sévit présentement en Allemagne sur ce sujet offre un exemple éloquent, mais c'est un exemple à ne pas suivre ${ }^{6}$. On trouve, d'un côté, des historiens qui voudraient que l'histoire nationale contribue à nourrir le fondement symbolique de la société et à favoriser l'intégration, ce qui me semble tout à fait légitime (je renvoie à ce que j'ai dit sur l'historisation des valeurs). Or, les tenants de cette idée sont assimilés à la pensée ethnique, c'est-à-dire à une pensée étroite, fermée au pluralisme. Ils sont aussi accusés de cultiver une vision utilitaire, instrumentalisée et donc révisionniste de l'histoire. Même si les travaux de certains de ces historiens versent dans les travers dont on les accuse, il me paraît abusif de condamner a priori le projet d'une mémoire soucieuse de contribuer (entre autres) à l'identitaire.

6. Pour un bon survol de cette controverse, voir Frédéric Boily, «Les enseignements de la "Querelle des historiens" ou sortir de l'histoire nationale au Québec», Bulletin d’histoire politique, 13, 2 (hiver 2005): 159-170. 
De l'autre côté, on trouve des partisans qui voudraient que l'histoire s'écrive à distance de l'identitaire et du national. La thèse prédominante, c'est que l'historien qui reste attaché au cadre national verse nécessairement dans la partisanerie, dans l'histoire romancée, dans la sélection délibérée des faits et des textes, en cachant «les faits gênants» et en rejetant la diversité des interprétations possibles. Moi, je dirais plutôt d'un historien qui travaillerait de cette façon qu'il est tout simplement un mauvais historien.

Comment une histoire aseptisée de cette manière pourrait-elle rendre compte des révolutions nationales qui ont secoué l'Europe à partir du début du XIX ${ }^{e}$ siècle et dont les réalisations sont indissociables de la mise en place des libertés civiles et des régimes démocratiques? Ou des mouvements de décolonisation des sociétés du Tiers monde, si étroitement associés à diverses formes de nationalismes? Ou, dans le cadre québécois, de la Conquête et des significations dont elle reste aujourd'hui lourdement chargée? Ou encore des luttes du mouvement patriote pour affranchir les Francophones du joug impérialiste?

Les partisans de cette histoire postnationale, expurgée, plaident aussi pour une approche "critique, différenciée et complexe». Mais ce sont là précisément les règles élémentaires de toute construction du passé, quelles qu'en soient les finalités. Les tenants d'une histoire postnationale affirment également que la nation ne doit pas être le seul prisme de l'écriture du passé. Mais qui voudrait être en désaccord avec cet énoncé? Il va de soi que la référence nationale doit toujours être croisée avec d'autres références: celle des classes sociales, des rapports de pouvoir, des inégalités, des rapports interculturels, de la vie symbolique, etc.

Je ne vois pas non plus en quoi une histoire qui serait consacrée à la promotion de la citoyenneté pourrait échapper au procès de «sens" et s'en tenir à l'écriture du passé «tel qu'il a été». Inévitablement, une telle histoire, comme toute entreprise historiographique, est destinée à promouvoir des valeurs, à prendre à témoin des épisodes, des acteurs - donc à sélectionner. Se mettant au service de la citoyenneté, elle aussi est menacée d'instrumentalisation. Et quoi qu'il en soit, elle a aussi le devoir de montrer que, pour ce qui est des $\mathrm{XIX}^{\mathrm{e}}$ et $\mathrm{XX}^{\mathrm{e}}$ siècles, le passé des sociétés occidentales s'est déroulé dans un cadre national, d'où la nécessité de rappeler comment les événements charnières y ont été vécus et les significations qu'ils ont portées. Enfin, cette histoire dite citoyenne ne peut pas non plus faire l'impasse sur les angoisses de chaque génération ni sur les idéaux qui ont motivé les grands mouvements politiques, cultu- 
rels et sociaux. Ce que je veux souligner ici, c'est que le civique est tout simplement indissociable du culturel.

Finalement, cette controverse tend à opposer une vision du passé «tel qu'il a été» à une vision du passé porteur de sens. Or, une telle antinomie relève d'une perception positiviste de la science historique. Depuis plusieurs décennies, on ne prétend plus écrire le passé «tel qu'il a été», on l'écrit toujours à partir de paradigmes, de questions, de repères théoriques plus ou moins élaborés et d'hypothèses; on le fait aussi à l'aide d'instruments imparfaits mais perfectibles, toujours limités, parfois irrémédiablement biaisés (les recensements, par exemple). Prétendre reconstituer le passé tel qu'il a été, c'est nous ramener à la vieille histoire événementielle de Langlois et Seignobos dont le sort a été réglé depuis longtemps.

Il est assez étonnant de voir resurgir ce débat aujourd'hui. Il est indéniable que l'historien construit son interprétation du passé, mais il ne s'ensuit pas qu'il le fasse d'une façon arbitraire ou discrétionnaire. Il doit se soumettre à une méthode et s'assurer que son interprétation s'accorde avec les données empiriques, dont la collecte est également soumise à des règles. Il doit aussi, comme nous le savons tous, se soumettre à la critique. C'est dans cette interaction entre les questions, les hypothèses, la méthode et le débat critique que se construisent les interprétations du passé.

Je pense que cette querelle illustre parfaitement les culs-de-sac où peuvent conduire les fausses antinomies, les polarisations abusives. Elle témoigne aussi d'une vision très négative, très pessimiste de la nation, perçue comme un lieu de dérives dont il faut se défaire au profit de solidarités mondialisées. Il est significatif que cette historiographie postnationale, qu'on pourrait qualifier de radicale, soit très influente en Allemagne, une nation qui a versé dans les pires extrêmes, qui est aux prises avec une mémoire accablante et qui est tentée de condamner la nation plutôt que les classes qui l'ont pervertie. Comme si la sortie vers la mondialisation était affranchie des rapports de force et des mécanismes de manipulation qui en viennent à trahir même les plus nobles idéaux. Par ailleurs, il n'est pas inutile de rappeler ici que les ultra-nationalismes qui ont mis l'Europe à feu et à sang n'ont pas sévi partout. Le procès de la nation devrait en tenir compte.

\section{POUR UNE HISTOIRE INTÉGRANTE}

Revenons au Québec. L’enseignement de l'histoire nationale devrait avoir pour objectif principal de raconter, pour les raviver, les valeurs communes à vocation civique : les expériences qui les ont fait naître, les divisions, les 
conflits qui ont jalonné leur parcours, les obstacles qu'elles ont dû surmonter, les acteurs, les institutions qui les ont soutenues, les trahisons qu'elles ont subies, les promesses qu'elles représentent pour l'avenir.

Se pose aussi le problème de la diversité ethnoculturelle. Ce passé où on peut lire la genèse des valeurs d'une société, c'est surtout, et inévitablement, celui de la majorité fondatrice qui y trouve une résonance immédiate. Mais qu'en est-il des minorités, des nouveaux venus? D’abord, je rappelle que les valeurs historisées évoquées plus haut doivent avoir une vocation civique, à portée universelle. La plupart des citoyens devraient donc pouvoir s'y retrouver. En plus, il faut aussi considérer que les nouveaux Québécois, de même que les membres des minorités, sont eux aussi porteurs d'un passé et des valeurs qu'il a forgées. Cet élément doit être pris en compte et doit donner lieu à un dialogue de façon à ce que la vision du passé puisse intégrer toute la richesse symbolique de notre société.

À ce propos, je réfère à deux études récentes qui ont livré des résultats assez spectaculaires ${ }^{7}$ : non seulement la grande majorité des immigrés adhèrent aux valeurs principales du Québec (liberté, égalité, démocratie...), mais ils le font parfois avec plus d'intensité que les membres de la société d'accueil. L'une des raisons est simple: plusieurs de ces immigrants ont quitté leur pays justement parce qu'ils étaient privés de ces valeurs 8 .

Il me semble impossible d'écrire l'histoire du Québec (ou de toute autre société d'Occident) en évacuant la question nationale. Que cela plaise ou non, c'est une trame qui traverse une très grande partie de notre histoire, qui a mobilisé la population de mille façons et qui l'interpelle encore fortement aujourd'hui.

Cela dit, je voudrais aussi nous mettre en garde - nous, de la majorité francophone - contre une façon d'écrire le passé québécois qui ne le rendrait accessible et significatif qu'à la majorité fondatrice. Ce serait une erreur coûteuse. Les grands épisodes de notre histoire, ses expériences déterminantes, peuvent tous se décliner sur le mode universel et solliciter

7. Il s'agit, en premier lieu, d'un sondage effectué à l'échelle canadienne par Environics Research Group, à l'initiative de la Fondation Trudeau et de l'Université Dalhousie en novembre 2011; ses résultats ont été dévoilés lors d'un colloque de la Fondation (17-19 novembre 2011) à Halifax. C'est, en deuxième lieu, une étude réalisée par Antoine Bilodeau, «Is Democracy the Only Game in Town? Tension Between Immigrants' Democratic Desires and Authoritarian Imprints", conférence présentée au colloque "The Political Incorporation of Immigrants: Progress, Prospects and Pitfalls in Europe and North America ", UC-Berkeley (4-5 mars 2011).

8. Voir sur ce sujet G. Bouchard, L'interculturalisme..., op. cit., 113-114. 
l'attention de très larges publics au-delà de nos frontières. Par exemple: comment se sont faites sur plus de trois siècles l'occupation et la mise en valeur du territoire; comment une minorité culturelle isolée sur le continent le plus puissant du monde a su non seulement survivre mais se développer d'une façon remarquable; comment se sont opérées chez nous la décolonisation et la construction d'un État libéral; comment se sont mises en place la démocratie, la modernité, la laïcité; comment et dans quelle mesure nous avons pu contrer les effets pervers du capitalisme; etc.

Raconter le passé québécois dans cette perspective, ce n’est pas le diluer ni le trahir, c'est au contraire intégrer sa singularité à un discours qui l'universalise et qui l'agrandit. Ce devrait être l'objectif de ce que j'appelle une histoire intégrante.

Je pense que l'histoire doit produire un savoir, incontestablement, mais elle doit aussi dégager un sens et une émotion. Le sens, c'est celui qui est véhiculé par les valeurs historisées à portée universelle. L'émotion, c’est celle qui émane des épisodes et des acteurs qui ont forgé et incarné ces valeurs - l'historien travaille toujours sur un matériau humain, quelle que soit la façon dont il est abordé. Dans toute société, une idée, une valeur acquiert une autorité, une capacité mobilisatrice à proportion de ce qu'elle s'enracine à la fois dans la raison et dans l'émotion. Ensemble, savoir, sens et émotion nourrissent à la fois l'identité et la citoyenneté. Il me semble que ces dimensions ne peuvent être dissociées si, encore une fois, on veut éviter des dichotomies qui, comme toutes les dichotomies, font obstacle à la réflexion plus qu'elles ne l'éclairent ${ }^{9}$.

\section{CONCLUSION : L'HISTOIRE COMME BOUILLON DE CULTURE}

Quelques remarques, pour conclure. D’abord, je pense que l'interculturalisme québécois offre une grille de pensée permettant de dépasser les fausses polarités qui divisent un grand nombre de sociétés contemporaines en matière de gestion de la diversité ethnoculturelle. C'est une pensée des équilibres, des médiations et des synthèses, hostile aux dichotomies, aux polarisations trompeuses. Cela dit, elle recherche non pas les compromis simplistes, à la Salomon, mais l'invention féconde de nouveaux arrangements en faisant appel à l'imagination créatrice, celle qui dénoue les impasses, crée des consensus et fait progresser.

9. Pour une critique de la pensée dichotomique, voir Gérard Bouchard, «Nation et co-intégration: contre la pensée dichotomique», dans J. Maclure, A.-G. Gagnon, dir., Repères en mutation. Identité et citoyenneté dans le Québec contemporain (Montréal, Québec Amérique, 2001), 21-36. 
Sur un autre plan, je ne crains pas d'adopter l'État-nation comme cadre de réflexion. Il faut se rappeler, par exemple, que tout ce qui concerne la gestion de la diversité ethnoculturelle, qu'il s'agisse de l'immigration, des modèles d'intégration, de l'application des droits, de la définition de l'identité, ou du régime de laïcité, tout cela relève des pouvoirs dévolus à l'État-nation qui reste donc un acteur primordial.

Néanmoins, selon certains, l'attention portée à l'État-nation irait à l'encontre de l'histoire qui se fait, elle serait à rebours des nouveaux horizons de pensée et d'action ouverts par la mondialisation. Or, je soutiens que c'est précisément à cause de la mondialisation qu'il faut plaider également pour la nation.

Qu'on me comprenne bien, je ne rejette pas la mondialisation, loin de là. Elle ouvre les horizons, elle rapproche les peuples, elle instaure des garde-fous contre les nations délinquantes, elle met en place de puissantes instances qui œuvrent à la diffusion de valeurs fondamentales, elle instaure des interdépendances salutaires. C'est aussi de là que sont venues les grandes campagnes pour la paix dans le monde, pour les droits humains, pour la préservation de la diversité des cultures, pour le développement durable, pour l'alphabétisation dans les pays démunis, pour la protection des droits de l'enfance, etc.

Mais la mondialisation a ses revers. Elle a donné lieu à la formation de puissants acteurs économiques et politiques qui ont déplacé les centres de décision en les éloignant des citoyens. Elle a été piégée par le néolibéralisme, par le tout-au-marché qui a fait croître presque partout les inégalités sociales, qui a affaibli les solidarités locales, qui a réduit le pouvoir des États et des parlements, qui a fait régresser les syndicats, qui a fait renaître dans les relations de travail des façons de faire qu'on croyait révolues depuis l'époque du capitalisme sauvage (par exemple, l'exploitation éhontée du travail des enfants). Elle a aussi, comme on sait, ouvert des perspectives inouïes à la corruption et à la fraude fiscale, dont les paradis fiscaux ne sont qu'une figure parmi d'autres.

Dans ce contexte, l'État-nation est en train de se redéfinir une autre fois, de s'ouvrir à une nouvelle vocation qui consiste à se poser comme refuge, comme écran protecteur de la démocratie et du citoyen contre les dangers, contre les effets aliénants de la mondialisation. L'État-nation peut et doit le faire en s'efforçant de maintenir les politiques sociales, en sauvegardant la démocratie, en protégeant les cultures nationales qui assurent aux citoyens un environnement symbolique dont se nourrissent les identités, en leur donnant le sentiment qu'ils peuvent encore agir sur leur 
société, qu'ils ont toujours une prise - si modeste soit-elle - sur leur destin. En d'autres mots, l'État-nation doit survivre comme contrepoids à une mondialisation dont le mouvement nous échappe, en particulier lorsqu'on est une petite nation.

Dans cette direction, le droit et la culture, loin de s'opposer, servent le même combat. L'histoire nationale également, suivant les voies que j'ai suggérées. Le danger ici-j'y reviens toujours - ce serait de rompre l'équilibre qui doit s'établir entre le cadre mondial et le cadre national. Cet équilibre peut se rompre de deux façons, toutes les deux aux dépens de la nation: soit qu'elle se livre aveuglément à la mondialisation, soit qu'elle $s^{\prime}$ installe dans des positions de repli.

La crise des accommodements qui a conduit à la création de la Commission que j'ai coprésidée avec Charles Taylor en $2007-2008^{10}$ était alimentée en bonne partie par l'insécurité collective, par la crainte de l'Autre, tout cela jumelé, bien sûr, avec une inflation médiatique que notre rapport a dénoncée. Mais ce dernier facteur ne doit pas faire oublier le coefficient d'insécurité qui préexistait dans un contexte marqué par la faible fécondité, le vieillissement de la population, l'affaiblissement de l'État comme levier collectif, l'augmentation du nombre des immigrants, la crainte du terrorisme islamiste et la nouvelle menace pour le français que constitue la mondialisation.

On se souvient que cette insécurité a été exploitée par le chef d'un parti politique de second rang (l’Action démocratique du Québec) qui a pu de cette façon former l'opposition à l'Assemblée nationale. Quant au gouvernement, il a laissé sans suite, au cours des années qui ont suivi, une grande partie des recommandations formulées dans le rapport de la Commission $^{11}$. En conséquence, l'insécurité est demeurée et elle vient d'être

10. Il s'agit de la Commission de consultation sur les pratiques d'accommodement reliées aux différences culturelles. Elle a déposé son rapport en mai 2008: Fonder l'avenir: Le temps de la conciliation, Gouvernement du Québec, 310 pages.

11. La Table de concertation des organismes au service des personnes réfugiées et immigrantes (TCRI) a dressé un bilan très sévère de l'action (ou inaction?) gouvernementale, estimant à $10 \%$ environ la proportion des recommandations ayant connu des suites (voir le «Bulletin de performance à l'endroit du gouvernement du Québec» émis le 12 avril 2010). L'exercice a été répété au printemps 2011 avec sensiblement les mêmes conclusions. Cette Table de concertation regroupe 139 organismes oeuvrant à travers le Québec (www.tcri.qc.ca). Le politologue François Rocher, de son côté, a établi cette proportion à $50 \%$ environ en s'appuyant sur une méthodologie sophistiquée («Effet miroir ou miroir aux alouettes? Bilan préliminaire de la mise en œuvre des recommandations de la Commission Bouchard-Taylor», communication présentée au congrès annuel de la Société québécoise de science politique, Université d'Ottawa, 23 mai 2012). Quoi qu'il en soit, il est certain que des recommandations parmi les plus importantes sont restées sans suite, celles, notamment, qui invitaient à la production de livres blancs sur la laïcité et sur l'interculturalisme. 
harnachée à nouveau par le gouvernement du Parti québécois qui est en place depuis septembre 2012 et qui, parlant au nom de «la majorité silencieuse", active les réflexes de peur.

Le débat ouvert par le projet de charte des valeurs a réveillé chez plusieurs Francophones québécois de vieux démons hérités d'une époque qu'on croyait révolue: à cause des pratiques d'accommodement en faveur des immigrants et des minorités (et, plus largement, en faveur des citoyens porteurs d'une différence religieuse), nos valeurs seraient bafouées, notre identité serait menacée, la séparation institutionnelle de l'État et des Églises serait en péril, nous ferions face, en somme, à une importante régression qui pourrait effacer tout le travail d'émancipation réalisé depuis la Révolution tranquille. Il faut, nous dit-on, «se tenir debout», "se défendre »: mais contre quoi, contre qui, sinon contre les éléments les plus fragiles de notre société?

Il y a ici également du travail pour la science historique: rappeler les moments sombres de notre histoire que notre société a pu surmonter grâce à son énergie, son audace, sa créativité; rappeler la diversité qui a toujours fait partie de notre paysage socioculturel et avec laquelle nous avons appris, tant bien que mal, à composer; rappeler qu'au milieu du XIX siècle, les Francophones et les Anglophones étaient pratiquement à égalité à Québec et à Montréal - les Francophones, aujourd'hui, ne représentent pas moins de $80 \%$ de la population et la francophonie québécoise est bien vivante; rappeler que l'histoire de cette francophonie est faite d'avancées et de reculs, d'ajustements, de réinventions et d'expansions par inclusion; rappeler aussi que la tentation du repli nous a toujours coûté très cher collectivement et que c'est en se projetant vers de nouveaux horizons que nous avons su nous développer.

En tant que productrice de savoir, de sens et d'émotion hostile aux fausses antinomies, la science historique, plus que jamais, demeure un lieu de rencontre et de rapprochement interculturel, un foyer d'échange et de réflexion sur le passé comme sur le présent, en même temps qu'une source de construction des valeurs et des idéaux que nous désirons promouvoir ensemble. Mais elle ne peut le faire que dans une quête des formules d'équilibres, en s'efforçant - pour ce qui concerne l'histoire nationale - de conjuguer le juridique et le symbolique, le civique et l'identitaire. 